International Journal of Biology, Pharmacy and Allied Sciences (IJBPAS) 'A Bridge Between Caboratoryand QRendo' WWw.ijbpas.com

\title{
TAXONOMIC INVENTORY BASED ON PHYSICAL DISTRIBUTION OF MACROFUNGI IN MT. MACULOT, CUENCA, BATANGAS, PHILIPPINES
}

\section{MINERVA C. ARENAS ${ }^{1 * \& 2}$, EDWIN R. TADIOSA ${ }^{3}$ AND RENATO G. REYES ${ }^{1 \& 4}$}

1: Biology Department, College of Science, De La Salle University, Taft Avenue, Manila, Philippines

2: Biology Department, Institute of Arts and Sciences, Far Eastern University, Nicanor Reyes St., Sampaloc , Manila,

3: Botany and National Herbarium Division, National Museum of Natural History, T.M Kalaw Ave., Ermita, Manila

4: Center for Tropical Mushroom Research and Development, Department of Biological Sciences

College of Arts and Sciences, Central Luzon University, Science City of Muñoz, Nueva Ecija

*Email for correspondence: minerva arenas@dlsu.edu.ph

Received 29 ${ }^{\text {th }}$ Dec. 2017; Revised $20^{\text {th }}$ Jan. 2018; Accepted $27^{\text {th }}$ January 2018; Available online $1^{\text {st }}$ May 2018

DOI: https://doi.org/10.31032/IJBPAS/2018/7.5.4420

\section{ABSTRACT}

This research was conducted to assess the macrofungi and their physical distribution in terms of habitat, collection time, and elevation at Mt. Maculot, Cuenca, Batangas, Philippines. Field sampling was done using five transect lines each with 1,000 meters long in five different habitats. Opportunistic sampling was made from a baseline of 200 masl (meter above sea level) to 950 masl high. The collection sites were surveyed in different months of 2017 , i.e. February, June, September and December. Collected macrofungal samples were categorized based on macro-anatomical characteristics. The documentation resulted in the identification of 92 species under 5 classes, 33 families, and 57 genera. Out of 92 collected taxa, $89 \%$ of collection were found inhabiting the forested area; $26 \%$ in grassland; $24 \%$ in shrub land / agricultural / denuded; $9 \%$ in rocky areas; and 11\% in cliff. As regards to the collection time, $59 \%$ of 92 taxa were documented in February, 58\% in June, 54\% in September and 63\% in December. Moreover, in accordance with elevation, 29\% out of 92 taxa were observed between 348 - 405(masl), 8\% of taxa in 406 - 463masl, 0 collection between 464 - 579 masl, $2 \%$ of taxa at $580-695$ masl, $36 \%$ of taxa in $696-753$ masl, $47 \%$ taxa in $754-811$ masl, $21 \%$ 
taxa in $812-869$ masl and $22 \%$ taxa in $870-927$ masl. The results suggest that the presence of macrofungal species is greatly affected by physical distribution.

Keywords: habitat, macrofungi, transect, physical distribution, Mt. Maculot

\section{INTRODUCTION}

Macrofungi are categorized as having spore-bearing fruiting bodies visible to the naked eye, which can be in the form of mushrooms, brackets, puffballs, falsetruffles, cup fungi, etc. $[1,2]$. They are the noteworthy component of the terrestrial ecosystem, which play crucial role in nutrient cycling [3]. Also have economical and medicinal importance, for they are good sources of food and nutraceutical products. These eukaryotic organisms are incapable of producing their own food, thus, they depend on the substrate for survival [4].

Globally there have been few studies on macrofungi, such as cited by Mueller [1] estimated 53,000 to 110,000 species. Hence, taxonomic studies of these organisms in the Philippines gains minimal attention, since there limited numbers of studies done in this field. Some of the areas surveyed are in Cavite [5], Aurora [6], and Batangas [7]. More studies are needed and should be made to account for the continuing survey of macrofungi in the Philippines.

Mt. Maculot is in the municipality of Cuenca in the province of Batangas, located in the Southern part of Luzon. It is between $13^{\circ} 55.241^{\prime} \mathrm{N}$ latitude and $121^{\circ} 02.513^{\prime} \mathrm{E}$ longitude and has an altitude of 200 to 963 masl $[8,9]$. The mountain is considered as one of the popular mountain ecotourism site in the Philippines. Around three hours drive from Manila with it's rocky and cliff areas make it interesting weekend tourist spot for climbers and Catholic devotees on a Holy week for its famous Grotto [9].

Based on the case study done in ecotourism, the average monthly visitors in Mt. Maculot are around 3,000 persons [10]. With this notable disturbance of tourist visiting the mountain, which is evidently crossing the different habitat in the area and a common knowledge that species distribution is greatly affected by forest fragmentation [11], and the idea that the vegetation in the mountain influence the abundance of macrofungal species in the place [12]. Therefore, the researchers decided to conduct a study on how the trampled different habitat such as forested, grassland, shrub, agricultural, denuded, rocky and cliff account for the presence of macrofungi in Mt. Maculot. Moreover, evaluations of macrofungal species presence and absence in four times collection and in different altitude were also noted and measured. 


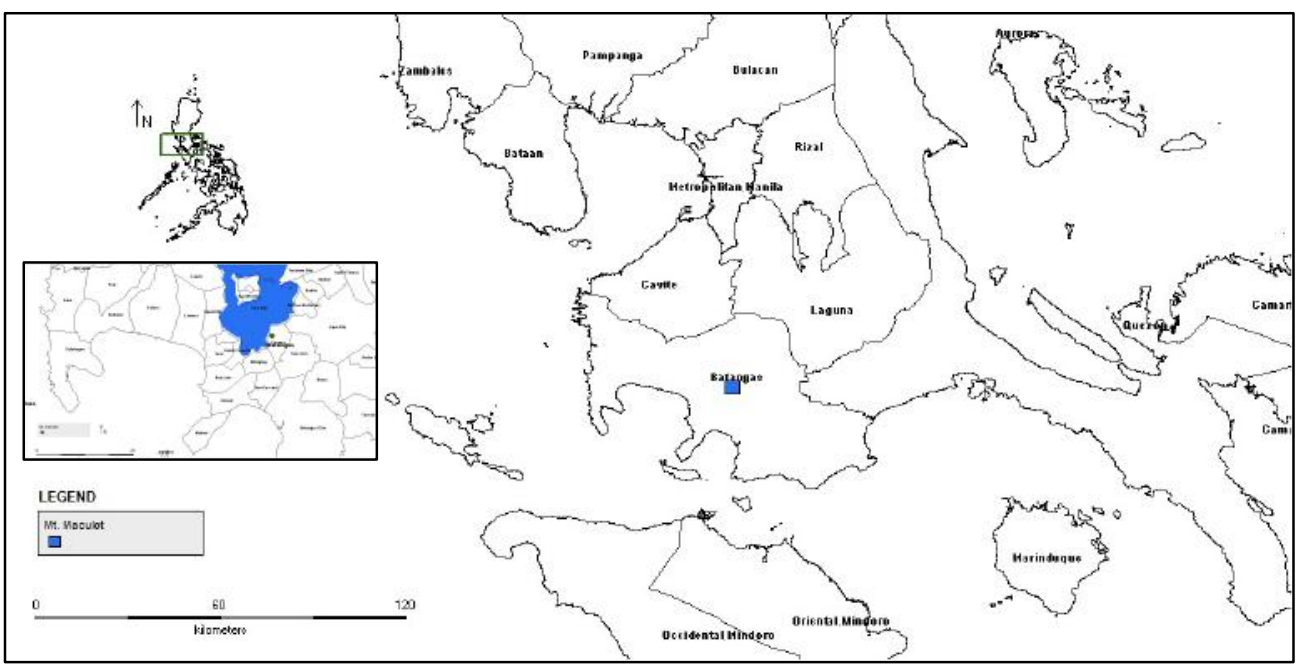

Fig.1 Map showing location of Mt. Maculot in the province of Batangas

\section{MATERIALS AND METHODS}

\section{Field sampling of macrofungal species}

Field sampling was accomplished using opportunistic sampling in different habitats such as forested area, grassland, shrub land / agricultural / denuded, rocky areas, and cliff. Collection and documentation were done in five transect lines each with 1000 meters long and started from a baseline of 200 masl up to 900 masl. The samplings were made in the months of February, June, September, and December. Substrates and growth habit of macrofungi, altitude of the site were recorded at the time of collection. Macrofungi that grow in soil were taken by trowel and those inhabiting the rotten tree logs were collected using a bolo.

\section{Identification of macrofungi}

The preliminary identification of collected samples was carried out using the taxonomic listing of Arenas [2], Lodge [12], Kuo [13], Ostry [14], Reyes [15], and
Tadiosa [16]. The final identification of collected samples was done at Philippine National Herbarium.

\section{Data Analysis}

In order to determine the distribution of macrofungi, percent composition was determined to compare the number of taxa existing in the different habitat, collection period and altitude.

\section{RESULTS}

\section{Taxonomic listing}

During the four months of sampling of macrofungi at Mt. Maculot, a total of 92 species under 5 classes, 33 families, and 57 genera, as presented in Figure 2 and Table 1 were recorded. The data gathered revealed that macrofungi belonging to Class Agaricomycetes are the most dominant in Mt. Maculot. Most of the collected specimens were classified under Family Polyporaceae (Figure 3). The macrofungi that were observed in rotten twigs, logs, and branches were mostly solitary to gregarious and few are resupinate (Table 2). 


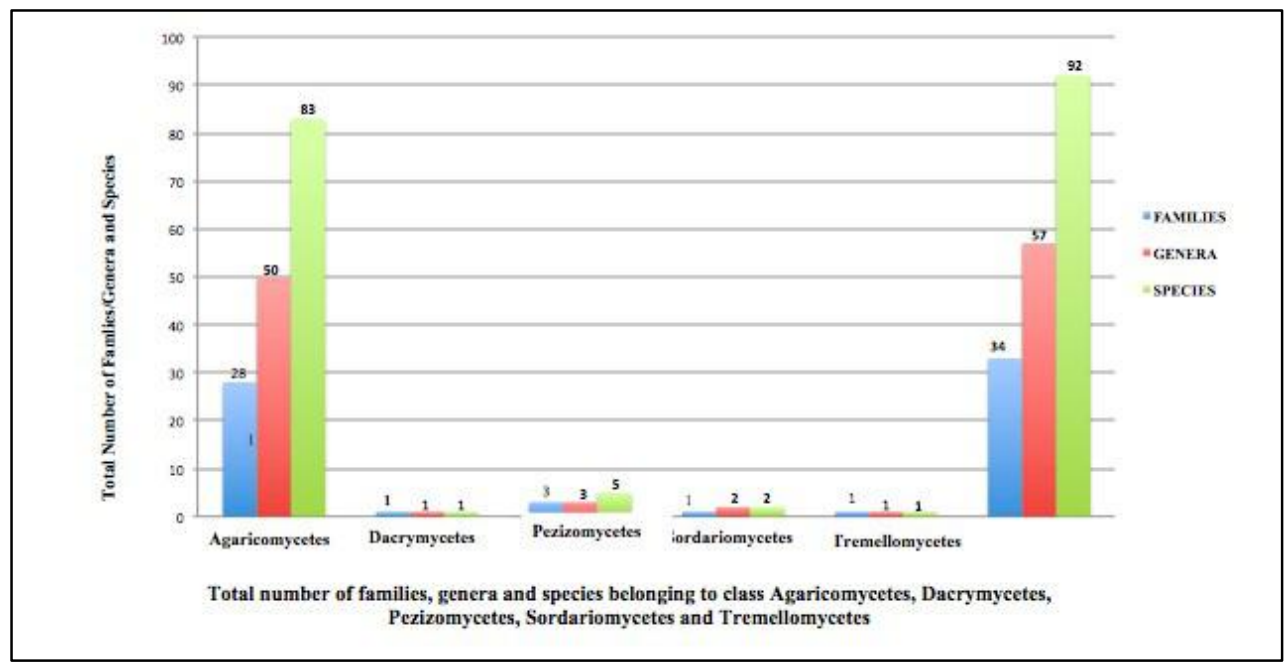

Figure 2: Classes of macrofungi collected in Mt. Maculot

Table 1: Taxonomic positions of the different macrofungi collected in Mt. Maculot

\begin{tabular}{|c|c|c|c|}
\hline & COPRDACFAF & Conrinur & \begin{tabular}{|l} 
Coprinus atramentarius (Bull.) Fr. \\
Conrinus comatus
\end{tabular} \\
\hline & |COPRINACEAE & Coprinus & Coprinus sp. \\
\hline & & $T_{1}$ & Lenzites $s p$. \\
\hline & Lanong & Lenzites & Lenzites striata (Swartz.) Fr. \\
\hline & |CUKIULACEAE & & Trametes corrugata (Pers.) Bres. \\
\hline & & Trametes & Trametes versicolor (L.) Lloyd. \\
\hline & & & Corticium confluens (Fr.) Fr. \\
\hline & CORTICIACEAE & Corticium & Corticium salmonicolor $\mathrm{B} . \& \mathrm{Br}$. \\
\hline & & & Corticium sp. \\
\hline & LODTDU & 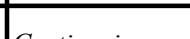 & Cortinarius callisteus (Fr.) Fr. \\
\hline & |CORIINARIACEAE & Cortinarius & Cortinarius sp. \\
\hline & CREPIDOTACEAE & Crepidotus & Crepidotus herbarum (Peck) Sacc. \\
\hline Agaricomycetes & & & Entoloma lividum (Bull.) Quelet. \\
\hline & |ENTOLOMATACEAE & Entoloma & Entoloma $\mathrm{sp}$. \\
\hline & L & $C_{-1}+l_{0}$ & Ganoderma applanatum (Pers.) Pat. \\
\hline & |GANODERMATACEAE & Ganoderma & Ganoderma lucidum (Leys.) Karst. \\
\hline & GEASTRACEAE & Geastrum & Geastrum triplex Jungh. \\
\hline & HYDNANGIACEAE & Laccaria & Laccaria sp. \\
\hline & & & Hygrocybe miniata (Fr.) Kumm. \\
\hline & LUVCPODUODACEAF & Hygrocybe & Hygrocybe sp. \\
\hline & |HYGROPHORACEAE & & Hygrophorus pratensis Fr. \\
\hline & & Hygropnorus & Hygrophorus sp. \\
\hline & & & Hymenochaete rubiginosa (Dick.) Lev. \\
\hline & HYMENOCHAETACEAE & Hymenochaete & Hymenochaete sp. \\
\hline & & Phellinus & Phellinus sp. \\
\hline & & & Marasmius ramealis (Bull.) Fr. \\
\hline & MADACMUACГАГ & Marasmius & Marasmius rotula (Scop.) Fr. \\
\hline & |MARASMIACEAE & & Marasmius sp. \\
\hline & & Omphalotus & Omphalotus sp. \\
\hline & MERULIACEAE & Cymatoderma & Cymatoderma elegans Jungh. \\
\hline & $\overline{\text { NIDULARIACEAE }}$ & Cyathus & Cyathus striatus Willd. \\
\hline & PHALLACEAE & Dictyophora & Dictyophora duplicata (Bosc.) E. Fisch. \\
\hline & PLUTEACEAE & Pluteus & Pluteus sp. \\
\hline & & Coriolus & Coriolus versicolor \\
\hline & & 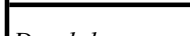 & Daedalea ambigua \\
\hline & & Daeaalea & Daedalea sp. \\
\hline & & & Fomes caryophylli (Rac.) Bres. \\
\hline & & Fomes & Fomes gilvus (Shwein.) Lloyd. \\
\hline & & & Fomes senex (Nees. \& Mont.) Cooke \\
\hline & & Microporus & Microporus xanthopus (Fr.) Kuntze \\
\hline & POLYPORACEAE & Panus & Panus rudis Fr. \\
\hline
\end{tabular}




\begin{tabular}{|c|c|c|c|}
\hline & & Coriolus & Coriolus versicolor (L.) Lloyd. \\
\hline & & & Daedalea ambigua Berk. \\
\hline & & Daeaalea & Daedalea sp. \\
\hline & & & Fomes caryophylli (Rac.) Bres. \\
\hline & & Fomes & Fomes gilvus (Shwein.) Lloyd. \\
\hline & & & Fomes senex (Nees. \& Mont.) Cooke \\
\hline & & Microporus & Microporus xanthopus (Fr.) Kuntze \\
\hline & POLYPORACEAE & Panus & Panus rudis Fr. \\
\hline & & & Polyporus grammocephalus Berk. \\
\hline & & & Polyporus hirsutus (Wulf.) Fr. \\
\hline & & Polyporus & Polyporus picipes Fr. \\
\hline & & & Polyporus pinsitus Fr. \\
\hline & & & Polyporus sp. \\
\hline & & Poria & Poria sp. \\
\hline & & Pycnoporus & Pycnoporus sanguineus Fr. \\
\hline & & & Lactarius piperatus (Scop.) Fr. \\
\hline & & Lactarius & Lactarius sp. \\
\hline & RUSSULACEAE & & Russula emetica Fr. \\
\hline & & | Kussuta & Russula sp. \\
\hline & SCHIZOPHYLLACEAE & Schizophyllum & Schizophyllum commune Fr. \\
\hline A cricomureter & STEPEACEAF & Storoum & Stereum ostrea (Bl.\& Nees.) Fr. \\
\hline Agaricomycetes & PIEKЕAСЕAЕ & Dtereum & Stereum sp. \\
\hline & & Stropharia & Stropharia rugosoannulata (Farlow) Murril \\
\hline & & Agrocybe & Agrocybe sp. \\
\hline & STROPHARACEAE & & Conocybe sp. \\
\hline & & Conocybe & Conocybe tenera (Schaeff.) Fayod \\
\hline & & Psilocybe & Psilocybe sp. \\
\hline & & Clitocybe & Clitocybe sp. \\
\hline & & Mycena & Mycena sp. \\
\hline & & Plourotus & Pleurotus ostreatus (Jacq. ex Fr.) Kummer \\
\hline & & Pleurotus & Pleurotus sp. \\
\hline & TRICHOLOMATACEAE & & Termitomyces albuminosa (Berk.) Heim \\
\hline & & Termitomyces & Termitomyces clypeatus R. Heim. \\
\hline & & & Termitomyces eurhizus (Berk.) R.Heim. \\
\hline & & 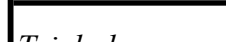 & Tricholoma saponaceum (Fr.) P.Kumm. \\
\hline & & Iricholoma & Tricholoma sp. \\
\hline & & & Cantharellus cibarius Fr. \\
\hline & CANTHARELLACEAE & Cantharellus & Cantharellus infundibuliformis (Scop.) Fr. \\
\hline & & & Cantharellus sp. \\
\hline & COPIOI $\triangle C F \triangle F$ & Howamani & Hexagonia apiaria (Pers.) Fr. \\
\hline & CORIULACEAE & Hexagonia & Hexagonia tenuis (Hook.) Fr. \\
\hline & HYDNACEAE & Hydnum & Hydnum sp. \\
\hline Dacrymycetes & DACRYOMYCETACEAE & Dacryopinax & Dacryopinax spathularia (Schwein.) Martin \\
\hline & LAPCOSOMATACБАГ & Galliela & Galiella rufa (Shwein.) Nannf.\& Korf. \\
\hline & DAKC USUIMA I ACEAE & Cookeina & Cookeina tricholoma (Mont.) Kuntze \\
\hline Pezizomycetes & PEZIZACEAE & Peziza & Peziza repanda Pers. \\
\hline & & & Octospora sp.1 \\
\hline & PYRONEMATACEAE & Octospora & Octospora sp.2 \\
\hline Sordariamustos & YYI $\triangle \mathrm{PI} A C E \wedge \mathrm{F}$ & Daldinia & Daldinia concentrica (Bolt.) Ces.\& de Not. \\
\hline Sordariomycetes & XYLARIACEAE & Xylaria & Xylaria polymorpha (Pers.) Grev. \\
\hline Tremellomycetes & TREMELLACEAE & Tremella & Tremella fuciformis Berk. \\
\hline
\end{tabular}


Table 2: Growth habits and substrates of collected macrofungi at Mt. Maculot

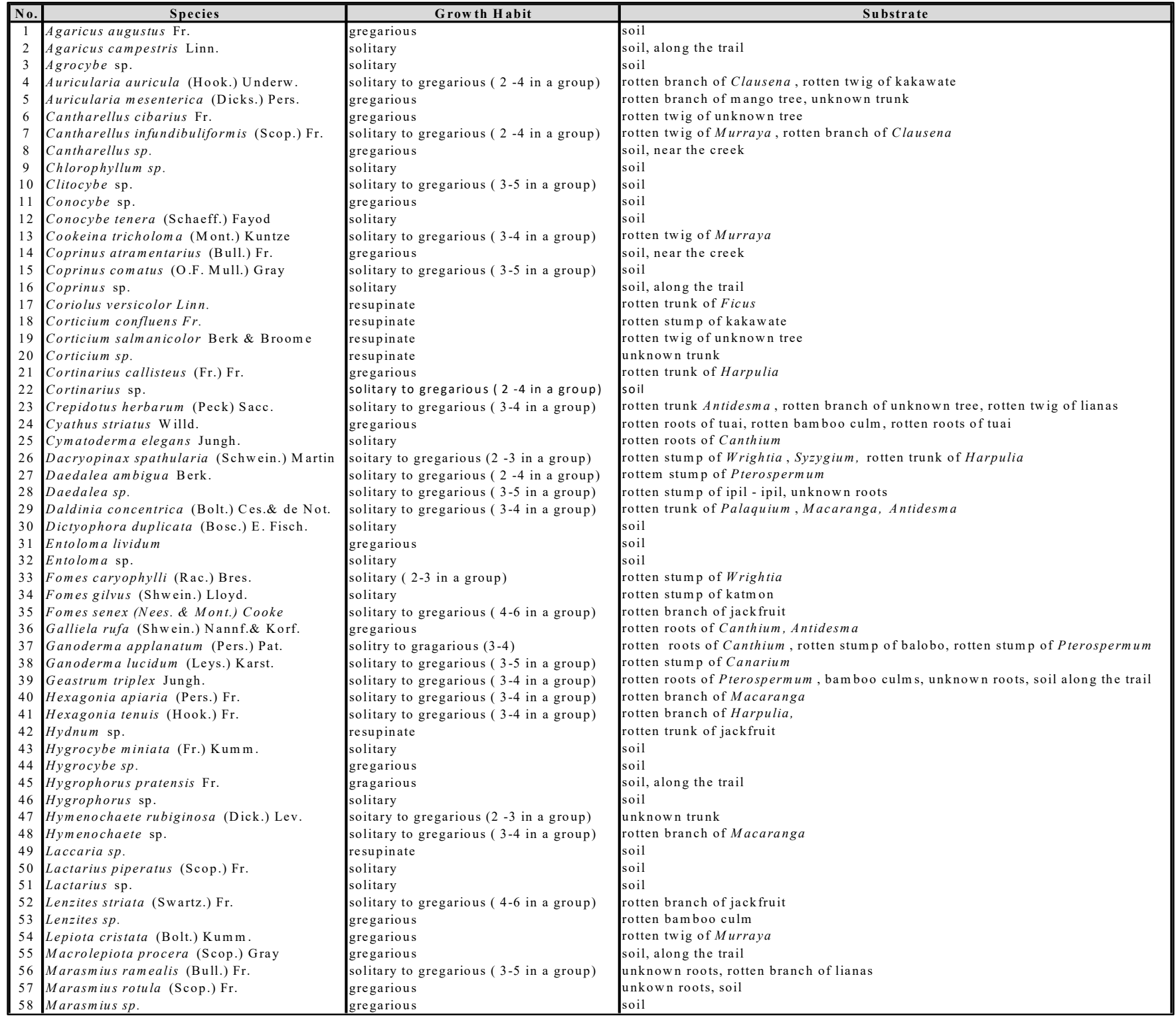

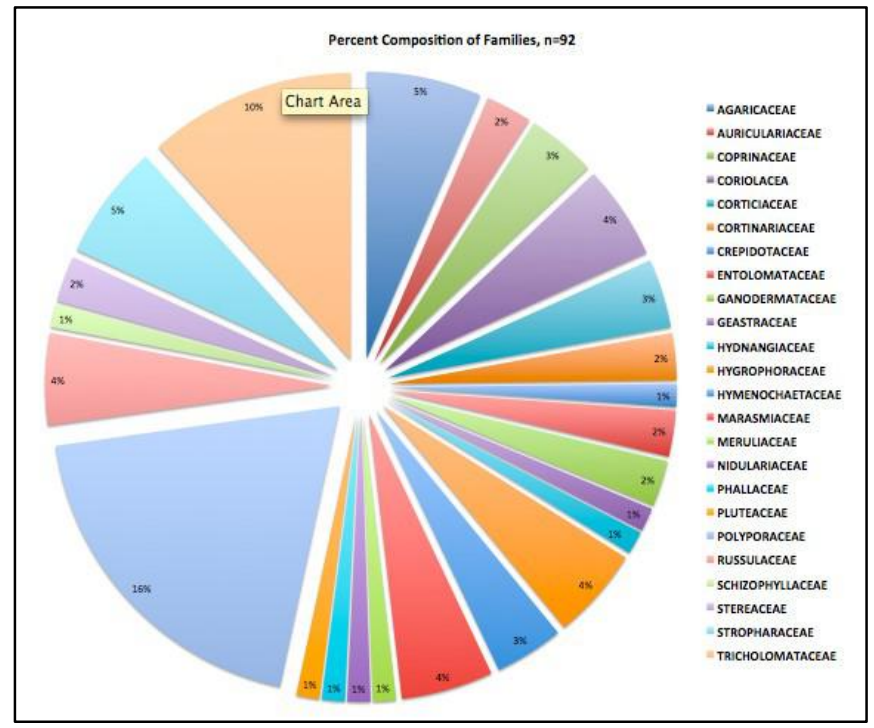

Figure 3: Percent composition of macrofungal families in Mt. Maculot 


\section{Physical Distribution of Macrofungi in}

\section{Mt. Maculot}

The climatic data at the time of collection are reflected in Table 3 wherein the highest temperature is $36.2^{\circ} \mathrm{C}$ in June and the lowest temperature is $17^{\circ} \mathrm{C}$ in February. On the other hand, relative humidity is highest in September and lowest in February. Moreover, amount of rainfall is highest $(326.8 \mathrm{~mm})$ in December, which corresponds to the number total of rainy days.

The macrofungal habitat distribution showed that among 92 taxa collected in Mt. Maculot, $82(89 \%)$ taxa were documented in forested area, $24(26 \%)$ taxa in grassland, $13(14 \%)$ taxa in shrub/agricultural/kaingin,
$8(9 \%)$ taxa in rocky and $10(11 \%)$ taxa in cliff as reflected in Table 4. In Table 5, the four months collection with 92 taxa 54 $(59 \%)$ found in the month of February, 53 $(58 \%)$ in June, 50 (54\%) in September and $58(63 \%)$ in December. Also, in Table 6 distribution of species in distinctive elevations are shown, $27(29 \%)$ of taxa were documented at 348 - 405masl, 9 $(10 \%)$ at $406-463$ masl, without collection at $464-579$ masl, $2(2 \%)$ of taxa at $580-$ 695masl, 33 (36\%) of taxa at 696-753masl, $43(47 \%)$ of taxa at $754-811$ masl, 19 $(21 \%)$ of taxa at $812-869$ masl, and 20 $(22 \%)$ of taxa at $870-927$ masl. The result showed that no common species were observed in all altitudes.

Table 3: Prevailing climatic conditions during collection

\begin{tabular}{|l|c|c|c|c|}
\hline \multirow{2}{*}{} & \multicolumn{4}{|c|}{ Ambulong Synaptic Station Data } \\
\cline { 2 - 5 } & February & June & September & December \\
\hline Highest Temperature $^{0} \mathrm{C}$ & 33.7 & 36.2 & 34.4 & 32.3 \\
\hline Lowest Temperature $^{0} \mathrm{C}$ & 17.6 & 23.5 & 21.2 & 21.1 \\
\hline Monthly Mean Relative Humidity $^{*}$ & 76 & 83 & 85 & 80 \\
\hline Monthly Rainfall (mm) & 0 & 78.9 & 321.15 & 326.8 \\
\hline Number of Rainy Days & 1 & 14 & 19 & 22 \\
\hline
\end{tabular}


Table 4: Distribution of macrofungi in different habitat at Mt. Maculot

\begin{tabular}{|c|c|c|c|c|c|c|}
\hline No. & \begin{tabular}{|l} 
Species \\
Agaricus augustus Fr.
\end{tabular} & \begin{tabular}{|l} 
Forested \\
\end{tabular} & Grassland & Shrub/Agricultural/Kaingin & Rocky & Cliff \\
\hline$\frac{1}{2}$ & $\begin{array}{l}\text { Agaricus augustus Fr. } \\
\text { Agaricus campestris Linn. }\end{array}$ & $\begin{array}{l}+ \\
+\end{array}$ & $\begin{array}{l}- \\
+\end{array}$ & 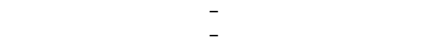 & - & - \\
\hline 3 & 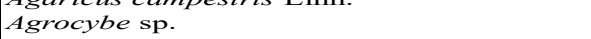 & + & + & & & \\
\hline 4 & Auricularia auricula (Hook.) Underw. & + & - & + & + & - \\
\hline 5 & Auricularia mesenterica (Dicks.) Pers. & + & + & & - & - \\
\hline 6 & Cantharellus cibarius Fr. & + & - & + & - & - \\
\hline 7 & Cantharellus infundibuliformis (Scop.) Fr. & + & - & - & - & - \\
\hline 8 & $\begin{array}{l}\text { Cantharellus sp. } \\
\text { Chlorophyllum sp. }\end{array}$ & + & - & - & - & - \\
\hline 10 & $\begin{array}{l}\text { Chlorophyllum sp. } \\
\text { Clitocybe sp. }\end{array}$ & + & + & - & - & - \\
\hline 11 & Conocybe sp. & + & + & - & - & - \\
\hline 12 & Conocybe tenera (Schaeff.) Fayod & + & + & - & - & - \\
\hline 13 & Cookeina tricholoma (Mont.) Kuntze & + & - & - & - & - \\
\hline 14 & Coprinus atramentarius (Bull.) Fr. & + & - & - & - & - \\
\hline 15 & Coprinus comatus (O.F. Mull.) Gray & + & - & - & - & - \\
\hline 16 & Coprinus sp. & + & - & - & - & - \\
\hline 17 & Coriolus versicolor Linn. & + & - & + & - & - \\
\hline 18 & Corticium confluens Fr: & + & - & - & - & - \\
\hline 19 & Corticium salmanicolor Berk \& Broome & + & - & - & - & - \\
\hline 20 & Corticium sp. & + & - & - & - & - \\
\hline 21 & Cortinarius callisteus (Fr.) Fr. & + & + & - & - & - \\
\hline 22 & Cortinarius sp. & + & - & - & - & - \\
\hline 23 & Crepidotus herbarum (Peck) Sacc. & + & - & - & - & + \\
\hline 24 & Cyathus striatus Willd. & + & - & + & - & - \\
\hline 25 & Cymatoderma elegans Jungh. & + & - & - & - & - \\
\hline 26 & Dacryopinax spathularia (Schwein.) Martin & + & - & + & - & - \\
\hline 27 & Daedalea ambigua Berk. & + & - & - & - & - \\
\hline 28 & Daedalea sp. & - & - & - & - & + \\
\hline 29 & Daldinia concentrica (Bolt.) Ces.\& de Not. & + & - & + & + & + \\
\hline 30 & Dictyophora duplicata (Bosc.) E. Fisch. & + & + & - & - & - \\
\hline 31 & Entoloma lividum & + & - & - & - & - \\
\hline 32 & Entoloma sp. & + & + & - & - & - \\
\hline 33 & Fomes caryophylli (Rac.) Bres. & + & - & - & - & - \\
\hline 34 & Fomes gilvus (Shwein.) Lloyd. & + & - & - & - & - \\
\hline 35 & Fomes senex (Nees. \& Mont.) Cooke & + & - & - & - & - \\
\hline 36 & Galliela rufa (Shwein.) Nannf.\& Korf. & + & - & + & - & - \\
\hline 37 & Ganoderma applanatum (Pers.) Pat. & + & - & + & - & - \\
\hline 38 & Ganoderma lucidum (Leys.) Karst. & + & - & - & - & - \\
\hline 39 & Geastrum triplex Jungh. & + & - & + & - & - \\
\hline 40 & Hexagonia apiaria (Pers.) Fr. & + & - & - & - & - \\
\hline 41 & Hexagonia tenuis (Hook.) Fr. & + & - & - & + & + \\
\hline 42 & Hydnum sp. & + & - & - & - & + \\
\hline 43 & Hygrocybe miniata (Fr.) Kumm. & - & + & - & - & - \\
\hline 44 & Hygrocybe sp. & + & + & - & - & - \\
\hline 45 & Hygrophorus pratensis Fr. & - & + & - & - & - \\
\hline 46 & Hygrophorus sp. & - & + & - & - & - \\
\hline 47 & Hymenochaete rubiginosa (Dick.) Lev. & + & - & - & - & - \\
\hline 48 & Hymenochaete sp. & + & - & - & - & - \\
\hline 49 & Laccaria sp. & + & - & - & - & - \\
\hline 50 & Lactarius piperatus (Scop.) Fr. & + & + & - & - & - \\
\hline 51 & Lactarius sp. & + & - & - & - & - \\
\hline 52 & Lenzites striata (Swartz.) Fr. & + & - & - & - & - \\
\hline 53 & Lenzites sp. & + & - & - & - & + \\
\hline 54 & Lepiota cristata (Bolt.) Kumm. & - & + & - & - & - \\
\hline 55 & Macrolepiota procera (Scop.) Gray & - & + & - & - & - \\
\hline 56 & Marasmius ramealis (Bull.) Fr. & + & + & - & - & - \\
\hline 57 & Marasmius rotula (Scop.) Fr. & + & + & - & - & - \\
\hline 58 & Marasmius sp. & + & - & - & - & - \\
\hline 59 & Microporus xanthopus (Fr.) Kuntze & + & - & - & + & + \\
\hline 60 & Mycena sp. & + & + & + & - & - \\
\hline 61 & Octospora sp.1 & + & - & - & - & \\
\hline 62 & Octospora sp.2 & + & + & - & - & - \\
\hline 63 & Omphalotus sp. & + & - & - & - & - \\
\hline 64 & Panus rudis Fr. & + & - & - & - & - \\
\hline 65 & Peziza repanda Pers. & + & - & - & - & - \\
\hline 66 & Phellinus sp. & + & - & - & - & - \\
\hline 67 & Pleurotus ostreatus (Jacq. ex Fr.) Kummer & + & - & + & - & - \\
\hline 68 & Pleurotus sp. & + & - & - & - & - \\
\hline 69 & Pluteus sp. & + & - & - & - & - \\
\hline 70 & Polyporus grammocephalus Berk. & + & - & - & - & - \\
\hline 71 & Polyporus hirsutus (Wulf.) Fr. & + & - & - & - & - \\
\hline 72 & Polyporus picipes Fr. & + & - & - & + & - \\
\hline 73 & Polyporus pinsitus Fr. & + & - & - & - & - \\
\hline 74 & Polyporus sp. & + & - & - & - & - \\
\hline 75 & Poria sp. & + & - & - & - & - \\
\hline 76 & Psilocybe sp. & + & - & - & - & - \\
\hline 77 & Pycnoporus sanguineus Fr. & + & - & - & + & - \\
\hline 78 & Russula emetica Fr. & + & + & & - & - \\
\hline 79 & Russula sp. & + & - & & - & - \\
\hline 80 & Schizophyllum commune Fr. & + & - & + & + & + \\
\hline 81 & Stereum ostrea (B1.\& Nees.) Fr. & + & - & - & - & - \\
\hline 82 & Stereum sp. & + & - & - & - & - \\
\hline 83 & Stropharia rugosoannulata (Farlow) Murril & - & + & - & - & - \\
\hline 84 & Termitomyces albuminosa (Berk.) Heim & + & - & + & - & - \\
\hline 85 & Termitomyces clypeatus R. Heim. & + & + & - & - & - \\
\hline 86 & s eurhizus (Berk.) R.Heim. & - & + & - & - & - \\
\hline 87 & Trametes corrugata (Pers.) Bres. & - & - & - & + & + \\
\hline 88 & Trametes versicolor (L.) Lloyd. & + & - & - & - & - \\
\hline 89 & Tremella fuciformis Berk. & + & - & - & - & - \\
\hline 90 & Tricholoma saponaceum (Fr.) P.Kumm. & + & - & - & - & - \\
\hline 91 & Tricholoma sp. & + & - & - & - & - \\
\hline 92 & Xylaria polymorpha (Pers.) Grev. & + & - & - & - & + \\
\hline & $\begin{array}{c}\text { Total number of taxa collected } \\
\end{array}$ & 82 & 24 & 13 & 8 & 10 \\
\hline
\end{tabular}


Table 5: Distribution of macrofungi in different time of collection at Mt. Maculot

\begin{tabular}{|c|c|c|c|c|c|}
\hline No- & $\begin{array}{r}\text { Species } \\
\text { Agaricus augustus Fr. }\end{array}$ & February & June & $\frac{\text { September }}{+}$ & \begin{tabular}{|l} 
December \\
-
\end{tabular} \\
\hline 2 & $\begin{array}{l}\text { Agaricus augustus Fr. } \\
\text { Agaricus campestris Linn. }\end{array}$ & - & $\overline{+}$ & + & $=$ \\
\hline 3 & Agrocybe sp. & + & - & + & - \\
\hline 4 & Auricularia auricula (Hook.) Underw. & + & + & + & - \\
\hline 5 & Auricularia mesenterica (Dicks.) Pers. & - & + & - & $\overline{+}$ \\
\hline 7 & Cantharellus infundibuliformis (Scop.) Fr. & + & + & + & + \\
\hline 8 & Cantharellus sp. & - & - & + & - \\
\hline 10 & $\begin{array}{l}\text { Chlorophyllum sp. } \\
\text { Clitocybe sp. }\end{array}$ & $\begin{array}{l}- \\
+\end{array}$ & - & $\stackrel{+}{+}$ & $\mp$ \\
\hline 11 & Conocybe sp. & - & - & - & + \\
\hline 12 & Conocybe tenera (Schaeff.) Fayod & + & - & - & + \\
\hline 13 & Cookeina tricholoma (Mont.) Kuntze & - & + & + & + \\
\hline 14 & Coprinus atramentarius (Bul1.) Fr. & - & + & - & + \\
\hline 15 & Coprinus comatus (O.F. Mull.) Gray & - & - & + & - \\
\hline 16 & Coprinus sp. & + & - & - & - \\
\hline 17 & Coriolus versicolor Linn. & - & - & - & + \\
\hline 18 & Corticium confluens Fr: & - & - & - & + \\
\hline 19 & Corticium salmanicolor Berk \& Broome & + & - & - & - \\
\hline 20 & Corticium sp. & - & + & - & - \\
\hline 21 & Cortinarius callisteus (Fr.) Fr. & + & + & + & + \\
\hline 22 & Cortinarius sp. & + & - & - & - \\
\hline $\begin{array}{l}23 \\
24\end{array}$ & Crepidotus herbarum (Peck) Sacc. & $\begin{array}{l}+ \\
+\end{array}$ & + & $\begin{array}{l}+ \\
+\end{array}$ & + \\
\hline 25 & $\begin{array}{l}\text { Cyathus striatus Willd. } \\
\text { Cymatoderma elegans Jungh. }\end{array}$ & + & + & + & + \\
\hline 26 & Dacryopinax spathularia (Schwein.) Martin & + & + & + & + \\
\hline 27 & Daedalea ambigua Berk. & + & + & - & + \\
\hline 28 & Daedalea sp. & - & - & + & \\
\hline 29 & Daldinia concentrica (Bolt.) Ces.\& de Not. & + & + & + & + \\
\hline 31 & Dictyophora duplicata (Bosc.) E. Fisch. & + & + & + & + \\
\hline 32 & $\begin{array}{l}\text { Entoloma lividum } \\
\text { Entoloma sp. }\end{array}$ & - & + & - & + \\
\hline 33 & Fomes caryophylli (Rac.) Bres. & - & - & - & + \\
\hline 34 & Fomes gilvus (Shwein.) Lloyd. & + & + & - & + \\
\hline 35 & Fomes senex (Nees. \& Mont.) Cooke & - & - & + & - \\
\hline 36 & Galliela rufa (Shwein.) Nannf.\& Korf. & - & + & + & + \\
\hline 37 & Ganoderma applanatum (Pers.) Pat. & + & + & + & + \\
\hline 38 & Ganoderma lucidum (Leys.) Karst. & & + & - & + \\
\hline 39 & Geastrum triplex Jungh. & + & + & + & + \\
\hline 40 & Hexagonia apiaria (Pers.) Fr. & - & + & & + \\
\hline 41 & Hexagonia tenuis (Hook.) Fr. & + & + & + & + \\
\hline 42 & Hydnum sp. & + & + & + & + \\
\hline 43 & Hygrocybe miniata (Fr.) Kumm. & - & + & - & + \\
\hline 44 & Hygrocybe sp. & + & - & + & - \\
\hline 45 & Hygrophorus pratensis Fr. & + & - & - & - \\
\hline 46 & Hygrophorus sp. & - & - & + & + \\
\hline 47 & Hymenochaete rubiginosa (Dick.) Lev. & - & - & + & + \\
\hline 48 & Hymenochaete sp. & + & - & + & - \\
\hline 49 & Laccaria sp. & - & - & + & - \\
\hline 50 & Lactarius piperatus (Scop.) Fr. & + & & + & - \\
\hline 51 & Lactarius sp. & + & - & - & + \\
\hline 52 & Lenzites striata (Swartz.) Fr. & + & + & - & + \\
\hline 53 & Lenzites sp. & + & - & + & \\
\hline 54 & Lepiota cristata (Bolt.) Kumm. & + & + & - & + \\
\hline 55 & Macrolepiota procera (Scop.) Gray & + & + & - & + \\
\hline 56 & Marasmius ramealis (Bull.) Fr. & + & + & + & + \\
\hline 57 & Marasmius rotula (Scop.) Fr. & + & + & + & + \\
\hline 58 & Marasmius sp. & + & + & - & - \\
\hline 59 & Microporus xanthopus (Fr.) Kuntze & + & + & + & + \\
\hline 60 & Mycena sp. & + & - & + & + \\
\hline 61 & Octospora sp. 1 & + & - & + & + \\
\hline 62 & Octospora sp.2 & + & - & + & + \\
\hline 63 & Omphalotus sp. & + & + & + & + \\
\hline 64 & Panus rudis Fr. & - & + & - & + \\
\hline 65 & Peziza repanda Pers. & - & + & + & + \\
\hline 66 & Phellinus sp. & + & + & - & + \\
\hline 67 & Pleurotus ostreatus (Jacq. ex Fr.) Kummer & - & + & - & + \\
\hline 68 & Pleurotus sp. & + & & + & - \\
\hline 69 & Plutens sp. & - & + & - & + \\
\hline 70 & Polyporus grammocephalus Berk. & & - & - & + \\
\hline 71 & Polyporus hirsutus (Wulf.) Fr. & - & + & - & + \\
\hline 72 & & + & + & + & + \\
\hline 73 & Polyporus pinsitus Fr. & - & + & - & + \\
\hline 74 & Polyporus sp. & + & & - & \\
\hline 75 & Poria sp. & + & + & + & + \\
\hline 76 & Psilocybe sp. & + & - & + & - \\
\hline 77 & Pycnoporus sanguineus Fr. & - & + & - & - \\
\hline 78 & Russula emetica Fr. & + & + & + & + \\
\hline 79 & Russula sp. & + & - & - & \\
\hline 80 & Schizophyllum commune Fr. & + & + & + & + \\
\hline 81 & Stereum ostrea (B1.\& Nees.) Fr. & & + & + & + \\
\hline 82 & Stereum sp. & + & - & - & - \\
\hline 83 & Stropharia rugosoannulata (Farlow) Murril & + & - & + & + \\
\hline 84 & Termitomyces albuminosa (Berk.) Heim & - & + & - & - \\
\hline 85 & Termitomyces clypeatus R. Heim. & + & - & + & + \\
\hline 86 & Termitomyces eurhizus (Berk.) R.Heim. & - & + & + & \\
\hline 87 & Trametes corrugata (Pers.) Bres. & + & - & + & + \\
\hline 88 & Trametes versicolor (L.) Lloyd. & + & - & - & - \\
\hline 89 & Tremella fuciformis Berk. & + & ( & - & ( \\
\hline 90 & Tricholoma saponaceum (I) & + & + & + & - \\
\hline 91 & Tricholoma sp. & 然 & + & - & - \\
\hline 92 & Xylaria polymort & + & + & - & + \\
\hline & Total number of taxa documented & 54 & 53 & $5 \%$ & 58 \\
\hline
\end{tabular}


Table 6: Distribution of macrofungi in different elevations

\begin{tabular}{|c|c|c|c|c|}
\hline No. & Species & $348-405$ & $406-463$ & $464-521$ \\
\hline 1 & Agaricus augustus Fr. & - & - & - \\
\hline 2 & Agaricus campestris Linn. & + & - & - \\
\hline 3 & Agrocybe sp. & + & - & - \\
\hline 4 & Auricularia auricula (Hook.) Underw. & + & - & - \\
\hline 5 & Auricularia mesenterica (Dicks.) Pers. & + & - & - \\
\hline 6 & Cantharellus cibarius Fr. & - & - & - \\
\hline 7 & Cantharellus infundibuliformis (Scop.) Fr. & + & + & - \\
\hline 8 & Cantharellus sp. & - & - & - \\
\hline 9 & Chlorophyllum sp. & - & - & - \\
\hline 10 & Clitocybe sp. & - & + & - \\
\hline 11 & Conocybe sp. & - & - & - \\
\hline 12 & Conocybe tenera (Schaeff.) Fayod & - & + & - \\
\hline 13 & Cookeina tricholoma (Mont.) Kuntze & - & - & - \\
\hline 14 & Coprinus atramentarius (Bull.) Fr. & - & - & - \\
\hline 15 & Coprinus comatus (O.F. Mul1.) Gray & - & - & - \\
\hline 16 & Coprinus sp. & - & - & - \\
\hline 17 & Coriolus versicolor Linn. & + & - & - \\
\hline 18 & Corticium confluens Fr. & - & - & - \\
\hline 19 & Corticium salmanicolor Berk \& Broome & - & - & - \\
\hline 20 & Corticium sp. & - & - & - \\
\hline 21 & Cortinarius callisteus (Fr.) Fr. & + & - & - \\
\hline 22 & Cortinarius sp. & - & - & - \\
\hline 23 & Crepidotus herbarum (Peck) Sacc. & - & - & - \\
\hline 24 & Cyathus striatus Willd. & + & - & - \\
\hline 25 & Cymatoderma elegans Jungh. & - & - & - \\
\hline 26 & Dacryopinax spathularia (Schwein.) Martin & - & - & - \\
\hline 27 & Daedalea ambigua Berk. & - & - & - \\
\hline 28 & Daedalea sp. & - & - & - \\
\hline 29 & Daldinia concentrica (Bolt.) Ces.\& de Not. & - & + & - \\
\hline 30 & Dictyophora duplicata (Bosc.) E. Fisch. & + & - & - \\
\hline 31 & Entoloma lividum & + & - & - \\
\hline 32 & Entoloma sp. & + & - & - \\
\hline 33 & Fomes caryophylli (Rac.) Bres. & - & - & - \\
\hline 34 & Fomes gilvus (Shwein.) Lloyd. & - & - & - \\
\hline 35 & Fomes senex (Nees. \& Mont.) Cooke & - & - & - \\
\hline 36 & Galliela rufa (Shwein.) Nannf.\& Korf. & + & - & - \\
\hline 37 & Ganoderma applanatum (Pers.) Pat. & + & - & - \\
\hline 38 & Ganoderma lucidum (Leys.) Karst. & - & - & - \\
\hline 39 & Geastrum triplex Jungh. & + & - & - \\
\hline 40 & Hexagonia apiaria (Pers.) Fr. & - & - & - \\
\hline 41 & Hexagonia tenuis (Hook.) Fr. & - & - & - \\
\hline 42 & Hydnum sp. & - & - & - \\
\hline 43 & Hygrocybe miniata (Fr.) Kumm. & + & - & - \\
\hline 44 & Hygrocybe sp. & + & - & - \\
\hline 45 & Hygrophorus pratensis Fr. & - & + & - \\
\hline 46 & Hygrophorus sp. & + & - & - \\
\hline 47 & Hymenochaete rubiginosa (Dick.) Lev. & - & - & - \\
\hline 48 & Hymenochaete sp. & - & - & - \\
\hline 49 & Laccaria sp. & - & - & - \\
\hline 50 & Lactarius piperatus (Scop.) Fr. & + & - & - \\
\hline 51 & Lactarius sp. & - & - & - \\
\hline 52 & Lenzites striata (Swartz.) Fr. & - & - & - \\
\hline 53 & Lenzites sp. & - & - & - \\
\hline 54 & Lepiota cristata (Bolt.) Kumm. & - & + & - \\
\hline 55 & Macrolepiota procera (Scop.) Gray & + & - & - \\
\hline 56 & Marasmius ramealis (Bul1.) Fr. & + & - & - \\
\hline 57 & Marasmius rotula (Scop.) Fr. & + & - & - \\
\hline 58 & Marasmius sp. & - & - & - \\
\hline 59 & Microporus xanthopus (Fr.) Kuntze & - & - & - \\
\hline 60 & Mycena sp. & + & - & - \\
\hline 61 & Octospora sp.1 & - & - & - \\
\hline 62 & Octospora sp. 2 & - & - & - \\
\hline 63 & Omphalotus sp. & + & - & - \\
\hline 64 & Panus rudis Fr. & - & - & - \\
\hline 65 & Peziza repanda Pers. & - & - & - \\
\hline 66 & Phellinus sp. & - & - & - \\
\hline 67 & Pleurotus ostreatus (Jacq. ex Fr.) Kummer & - & - & - \\
\hline 68 & Pleurotus sp. & - & - & - \\
\hline 69 & Pluteus sp. & - & - & - \\
\hline 70 & Polyporus grammocephalus Berk. & - & - & - \\
\hline 71 & Polyporus hirsutus (Wulf.) Fr. & - & - & - \\
\hline 72 & Polyporus picipes Fr. & - & - & - \\
\hline 73 & Polyporus pinsitus Fr. & - & - & - \\
\hline 74 & Polyporus sp. & - & - & - \\
\hline 75 & Poria sp. & - & - & - \\
\hline 76 & Psilocybe sp. & - & - & - \\
\hline 77 & Pycnoporus sanguineus Fr. & - & - & - \\
\hline 78 & Russula emetica Fr. & - & + & - \\
\hline 79 & Russula sp. & - & - & - \\
\hline 80 & Schizophyllum commune Fr. & + & - & - \\
\hline 81 & Stereum ostrea (B1.\& Nees.) Fr. & - & - & - \\
\hline 82 & Stereum sp. & - & - & - \\
\hline 83 & Stropharia rugosoannulata (Farlow) Murril & - & + & - \\
\hline 84 & Termitomyces albuminosa (Berk.) Heim & + & - & - \\
\hline 85 & Termitomyces clypeatus R. Heim. & + & - & - \\
\hline 86 & Termitomyces eurhizus (Berk.) R.Heim. & + & - & - \\
\hline 87 & Trametes corrugata (Pers.) Bres. & - & - & - \\
\hline 88 & Trametes versicolor (L.) Lloyd. & - & - & - \\
\hline 89 & Tremella fuciformis Berk. & - & - & - \\
\hline 90 & Tricholoma saponaceum (Fr.) P.Kumm. & - & - & - \\
\hline 91 & Tricholoma sp. & - & - & - \\
\hline 92 & Xylaria polymorpha (Pers.) Grev. & - & - & - \\
\hline & Total number of taxa documented & 27 & 9 & $\mathbf{o}$ \\
\hline
\end{tabular}




\section{DISCUSSION}

Members of the Order Agaricales are the most abundant among the macrofungi under Phylum Basidiomycota, in fact, onefifth of all the described fungi is contained in this group [17] and they propagate in a various habitat [18], hence their existence can be a symbiont, saprophytes and parasitic [4]. Thus, the richness of class Agaricomycetes in Mt. Maculot is due to the availability of numerous substrates in the area, making macrofungal species survived although it is one of the most visited mountains in the region[19].

The dominance of family Polyporaceae at Mt. Maculot is viable because the tree species in the mountain is diverse and $22.9 \%$ of them are even endemic [8], so more wood-rotting macrofungi can be seen in the forested area compared to other habitats. A study in the tree and macrofungal species revealed that the presence of tree, is positively correlated with fungal diversity [12]. In fact, in the study of Marzuki [20] 19 species of macrofungi were documented in a wood substrate which is distributed in the different designated stations. Therefore, forested area has more number of macrofungal species since it is undisturbed [25], which corresponds to the high occurrence of 82 taxa out of 92 taxa documented in the forested area at $\mathrm{Mt}$.
Maculot. Additionally, plant host specificity of wood rotting fungi has great effect on the distribution of macrofungal species in the forest [11].The host specificity is also evident in the result of the study of Pradhan [26] showing macrofungal specificity in terms of habitat such as natural forest, plantation forest, and villages.

Understanding the distribution of species in the habitat is important in the assessment of species in a particular place and time [4]. This is supported by the data collated in this study that shows a distinct number of macrofungal taxa wherein 82 out of 92 documented taxa. The other habitat which is basically grassland yielded poor number of documented taxa due to limited availability of substrates for macrofungi to grow. On the other hand, agricultural area is disturbed by residents due to intensive farming activities.

The Philippines has four types of climate being specific to each region. Mt. Maculot is in the eastern part of Batangas which falls under Type III climatic type, wherein there is no distinct maximum rain and has a short dry season i.e. from December to February or March to May [19]. Rainfall is one of the important factors to consider in macrofungal growth, in fact, many species emerged after rainfall within 10,15 , or 30 days [29] and the amount of 
rainfall which yielded abundant growth of macrofungi is $200 \mathrm{~mm}$. Another factor is the effect of the temperature on nutrition of fungi [13], a high occurrence is in the average of $23 \sim 24.9^{\circ} \mathrm{C}, \quad$ maximum of $28 \sim 31.9^{\circ} \mathrm{C}$, and minimum temperatures of $22 \sim 23.9^{\circ} \mathrm{C}$. The relative humidity is an additional factor for ample growth of fungi and the ideal is $>82 \%$ [14].

In another study in the course of temperature and relative humidity in mushroom cultivation, productive growth is in the range of $18 / 25^{\circ} \mathrm{C}-27 / 29^{\circ} \mathrm{C}$ and 55 $85 / 78-93 \%$ relative humidity [18]. Nowadays, fungal growth and distribution are affected by the concurrent climate change which is an inevitable fact that resulted in the disturbance of seasonal occurrence of fungal species [5],[24],[26]. The climatic data during the collection is within the range that stimulated the emergence of various macrofungal species. Moreover, the capacity of spores to store water and survived until enough moisture is absorbed in the environment, facilitated hyphal growth and eventually towards the development of fruiting bodies [15]. Thus, moisture and temperature have great influence on fungal growth in a favorable climatic condition [33].

The elevation or altitude is expressed as meters above sea level (masl), likewise a contributing factor in the distribution of macrofungi in the study site [16]. In this study, most macrofungi were documented at $696-753$ masl and in $754-811$ masl than in lower elevation. This is due to the availability of tree species as substrates in the forested area in high elevation [3]. And the number decreases at $812-927$ masl, this fact is conceivable because the Mt. Maculot Rockies is overseeing the Taal Lake and Taal Volcano, due to its well-known trail with well developed camp site for tourists, thus, many hikers often visit the mountain which causes stress to the vegetation and other inhabitants including macrofungi. In addition, sightseers may not be concerned in the conservation of Mt. Maculot to provide sustainability [7].

\section{CONCLUSION}

The collection yielded a total of 92 species which were grouped under five classes, the Agaricomycetes with 28 families, 50 genera, and 83 species; Dacryomycetes with one family, one genus and one species; Pezizomycetes with three families, three genera and five species; Sordariomycetes with one family, two genera and two species; and Tremellomycetes with one family, one genus and one species. The distribution of macrofungal species in the different habitat of Mt. Maculot implied that the forested areas with diverse tree species and is rarely visited by hikers contained the largest number of collected taxa compared with the cliff and rocky sites that have the least 
number of documented taxa. In the lowest elevation at 348masl - 405masl, the documented taxa are mostly gilled mushrooms that grow on leaf litter and in the soil. On the other hand, most macrofungal species in Mt. Maculot are confined in higher elevation various tree species. Despite being visited by hikers, Mt. Maculot still harbors different macrofungal species due to the availability of substrates such as rotting trees and leaf litters. However, there is still a need of continuously monitoring the macrofungal and other species for sustainability and conservation of Mt. Maculot.

\section{ACKNOWLEDGMENT}

The researchers conveyed their gratitude to the following individuals who assisted in the accomplishment of this study. The primary author's parents, Mr. and Mrs. Lawrence Fields, Superintendent of Taal Volcano Protected Landscape Area, Mr. Vic Mercado, Honorable Mayor Celerino Andaya of Cuenca, Batangas, Honorable Barangay Captain Celestino A. Lunar of Barangay Pinagkaisahan, field guide, Mr. Demetrio A. Magpantay, Mr. Abdullaziz Sam -Arcillo and Dr. Sandra L. Yap.

\section{REFERENCES}

[1] Chang, S.T. \& Buswell, J.A. Mushroom nutraceuticals. World Journal Microbiology

Biotechnology, 1996; 12: 473 -6
[2] Mueller, G. M., Schmit, J. P., Leacock, P. R. et al. Global diversity and distribution of macrofungi. Biodivers Conserv, 2007; 16(1), 37-48.

[3] Angelini, P., Bistocchi G., Arcangeli, A., Bricchi, E., Venanzoni, R. Divesity and Ecological Distribution of Macrofungi in a site of Community Importance of Umbria (Central Italy). The Open Ecology Journal, 2015; $8,1-8$.

[4] Reyes, R.G., Eguchi, F., Kalaw, S.P. and T. Kikukawa. Mushroom Growing in the Tropics: A Practical Guide. Nueva Ecija, Philippines. Central Luzon State University Press. 2009.

[5] Arenas, M. C., Tadiosa, E. R., Alejandro, G. J., Reyes, R. G. Macroscopic Fungal Flora of Mts. Palaypalay - Mataas na Gulod Protected Landscape, Southern Luzon, Philippines. Asian Journal of Biodiversity. 2015. 6 (1). 1 -22.

[6] Tadiosa, E.R., Agbayani, E.S. \& Agustin, N.T. Preliminary Study on Macrofungi of Bazal - Baubo Watershed, Aurora Province. Central Luzon, Philippines. Asian Journal of Biodiversity. 2011; 96, $149-171$.

[7] Tadiosa, E.R., R.U Briones. Fungi 
of Taal Volcano Protected

Landscape, Southern Luzon,

Philippines. Asian Journal of

Biodiversity. 2013;4: 46-64

[8] Arsenio, J., Medecilo, M. Mercado, E.,Salibay, E.Jr., Velera, F. A. Vegetation Analysis of Mt.

Maculot, Cuenca, Batangas, Philippines. International Conference on Environment and Industrial Innovation.2011;vol.12

[9] Dalisay, V.J. C. Ecotourism Development of Mt. Maculot in Cuenca Batangas, a case study. National and Regional Planning and Management.2016pp.1 -13.

[10] Berglund,H.,Johnson,B.G. Nested Plant and Fungal Communities; the importance of area and habitat quality in maximizing species capture in boreal old-growth forests. Biological Conservation.2003.112 pp.319328

[11] Lodge, D.J., Ammiranti, J.F., O’dell, T.E. \& Mueller, G. M. Collecting and describing macrofungi. In:Biodiversity of Fungi:Inventory Monitoring Methods (eds GM Mueller, GF Bills, MS Foster). Elsevier Academic Press, USA, 2004;128-158.
[12] Kuo, M. Mushroom: The big picture. 2011; Retrieved December 09, 2013 from the Mushroom Expert. Com Website: http://www.mushroomexpert.com/t axonomy. html.

[13] Ostry, M.E., Anderson, N.A. \& O'Brien, J.G. Field Guide to Common Macrofungi in Eastern Forests and Their Ecosystem Functions.2011.

[14] Reyes, R.G., Abella, E.A. \& Quimio T.H. Wild macrofungi of CLSU. Journal of Tropical Biology. 2003; 2, 8- 11.

[15] Tadiosa, E.R., Arenas, M.C., Reyes, R.G. Macroscopic Fungi of Mts. Banahaw - San Cristobal Protected Landscape Northwestern side, with a description of Nidula banahawensis sp.nov. (Basidiomycota). Asian Journal of Biodiversity.2015; Vol. 6 (2). 69 88.

[16] Kirk, P.M., Cannon, P, \& Stalpers., J (eds) Dictionary of the fungi, 10th edition. CABI, Wallingford. 2008.

[17] Paquit, J.C., Pampolina, N.M. Tree and Macrofungal diversity of the two different habitat types in Mt. Makiling forest reserve. International Journal of Microbiology and Mycology. 2017; 
Vol. 10 (4). 1 -8.

[18] Marzuki, B.M., Rossiana, N., \& Normita. Diversity of Macrofungi on Wood in Forest Nature Reserve of Bojonglarang Jayanti Cianjur West Java. Journal of Bacteriology and Mycology. 2017; Vol. 4(1).15.

[19] Gilbet, G.S., Gorospe, J. \& Ryvarden, L. Host and habitat preferences of polypore fungi in Micronesia tropical flooded forests. Mycological Research. 2008; 112674-680

[20] Pradhan, P. \& Acharya, K. Impact of Climate Change on the Diversity and Distribution of Fungal Community. Climate Change: Man and Environment. Chapter 8. Daya Publishing House, New Delhi. 2012; pp. 111120

[21] Lantican, R.M. The Science and Practice of Crop Production. College, Los Banos, Laguna, Phils.: SEAMEO SEARCA and UPLB. 2001;p 330

[22] Salerni,E., Laganà, A., Perini, C., Loppi, S., \& De Dominicis, V. Effect temperature and rainfall on macrofungi in oak forests of the Mediterrean area. Israel Journal of Plant Sciences. 2002; Vol. 50. Pp.189-198.
[23] Hoa, H.T., \& Wang C.L. The effects of temperature and nutritional conditions on mycelium growth of two oyster mushrooms (Pleurotus ostreatus and Pleurotus cystidiosus). Mycobiology.2015; 43(1):14-23.

[24] Hemmes, H.T., \& Wang C.L.The effects of temperature and nutritional conditions on mycelium growth of two oyster mushrooms (Pleurotus ostreatus and Pleurotus cystidiosus). Mycobiology. 2015; 43(1): 14-23.

[25] Kivaisi,A.K., Magingo, F.S.S. \& Mamiro, B. Performance of Pleurotus flabellatus on water hyacinth (Eichhornia crassipes) shoots at the different temperatures and relative humidity regimes. Tazmania Journal of Science. 2003; Vol 29 (2).

[26] Jang, S. K., \& Hur, T.C. Relationship between Climatic Factors Distribution of Higher Fungi in Byeonsbando National Park, Korea. Mycobiology. 2014; 42(1): 27-33.

[27] Dwivedi, Sandhya, Pampolina, Singh, Surendra, UK, Chauhan and Tiwari, Mahendra Kumar. Biodiversity Studies on Macro Fungi with Special Reference to Order Agaricales: Indian Scenario. 
Journal of Bacteriology \& Mycology. 2017; Vol 5(6). 1-4

[28] Boddy, L., Buntgen, U., Egli,S., Gange, A.C., Heegaard, E., Kirk, P.M., Mohammad, A., \& Kauserud, H. Climate variation effects on fungal fruiting. Fungal Ecology. 20113; 1-14

[29] Kauserud, H., Heegaard, R.H., Boddy, L., Hoiland, K., \& Stenseth, N. C. Mushroom's spore size and time of fruiting are strongly related: is moisture important?. Biology Letters. 2011; 7, $273-276$.

[30] Tally, S.M., Coley, P.D., \& Kursur, T.A. The effects of weather on fungal abundance and richness among 25 communities in the Intermountain West. BMC Ecology. 2002;2:7

[31] O'Dell, T.E., Ammirati, J.F. and Schreiner, E.G. Species richness and abundance of ectomycorrhizal basidiomycete sporocarps on a moisture gradient in the Tsuga heterophylla zone. Can. J. Bot. 1999;77: 1699-1711

[32] Kinge, T.R., Apalah, N.A., Nji, T.M., Acha, A.N. \& Mih, A. M. Species Richness and Traditional Knowledge of Macrofungi (Mushrooms) in the Awing Forest
Reserve and Community Northwest Region, Cameroon. Journal of Mycology.2017; pp 19.

[33] Castillo, R.C., Dimaano, P.D.C., Macaspac, E.A., Asilo, N.A. \& Amaro, J.C. Ecotourism in Mt. Maculot as assessed by the Host Community: Basis for Sustainable Development. International Journal of Sciences:Basic and applied research.2014; vol 15(1). 416-428. 